the interface at the level of single atoms and can therefore be tuned by controlling the details of the interface. A combination of pulsed-laser deposition and an in situ analysis technique, such as reflection high-energy electron diffraction, can provide the level of control needed to grow oxide heterostructures with atomically sharp interfaces ${ }^{3}$.

The work by Levy and co-workers relies on previous work that showed that the conductivity of the interface between these two oxides could be tuned. In 2006 the Augsburg team grew different numbers of lanthanum aluminate layers on a $\mathrm{TiO}_{2}$-terminated strontium titanate substrate and showed that there is an abrupt transition from insulating to metallic behaviour when the thickness of the lanthanum aluminate layer exceeds four unit cells ${ }^{4}$. This forms the basis of the writing and erasing of nanostructures demonstrated by the Pittsburgh-Augsburg team. Also in 2006, the present authors and co-workers in Twente and Antwerp ${ }^{5}$ found that if two closely spaced complementary interfaces $\left(\mathrm{LaO} / \mathrm{TiO}_{2}\right.$ and $\left.\mathrm{AlO}_{2} / \mathrm{SrO}\right)$ are present, they will be electronically coupled. When the distance between the interfaces was less than six perovskite-unit-cell layers (about $2.3 \mathrm{~nm}$ ), the interface conductivity and carrier density fell, although the carrier mobilities for the separate conducting interfaces remained high $\left(\sim 1,000 \mathrm{~cm}^{2} \mathrm{~V}^{-1} \mathrm{~s}^{-1}\right.$ at low temperatures) even when the spacing between the interfaces was less than $1 \mathrm{~nm}$.

The challenge to researchers was to exploit this ability to create active devices. Levy and co-workers used a conducting atomic force microscope to change the active surface layer (which is made of lanthanum aluminate) in their devices and thereby modify the conducting properties of the interface at the nanoscale ${ }^{6}$. The manipulation of ions, vacancies and so forth to influence the electronic properties of a nanoelectronic device in this approach can be compared with the manipulation of individual atoms and molecules on surfaces with various types of scanning probe microscopes ${ }^{7}$. However, the approach taken by Levy and co-workers has the advantage that it works at room temperature.

By making technologically important devices, such as field-effect transistors, Levy and co-workers have provided another example of the possibilities offered by the combination of new materials and the tools of nanotechnology. The approach is obviously capable of achieving very high integration densities and low power consumption. However, to be successful, any new approach must be able to improve the performance of current CMOS technology, especially in terms of device scalability, fabrication reproducibility, reliability and component lifetime. Our message to the oxide community is to build on these results and develop new kinds of devices that are capable of rising to this challenge.

Dave H. A. Blank and Guus Rijnders are at the $M E S A^{+}$Institute for Nanotechnology, University of Twente, 7500 AE Enschede, The Netherlands. e-mail:D.H.A.Blank@utwente.nl

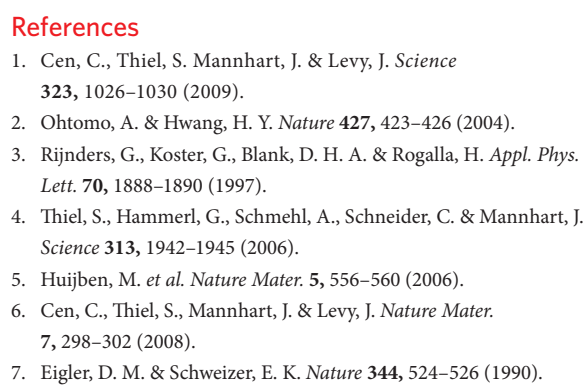

\title{
NANOPATTERNING
}

\section{What diffraction limit?}

It is widely assumed that diffraction places a limit on the smallest features that can be produced by light-based fabrication techniques, such as photolithography. This limit is typically about one-half to one-quarter of the wavelength of light involved. Now three independent groups of researchers have shown that it is possible to shatter this diffraction limit by using two light sources for photolithography.

A group at MIT (Science doi: 10.1126/ science.1167704; 2009) covered the surface they wanted to pattern with a film of photochromic molecules that becomes transparent when exposed to the light source with the shorter wavelength $(325 \mathrm{~nm})$ and transparent when exposed to the longer wavelength $(633 \mathrm{~nm})$. When exposed to both wavelengths the film is opaque, apart from small regions where the 325-nm light can pass through to produce structures with features as small as $36 \mathrm{~nm}$. This scanning electron micrograph shows trenches with a width of $94 \mathrm{~nm}$.

In the other experiments, a group at the University of Colorado in Boulder

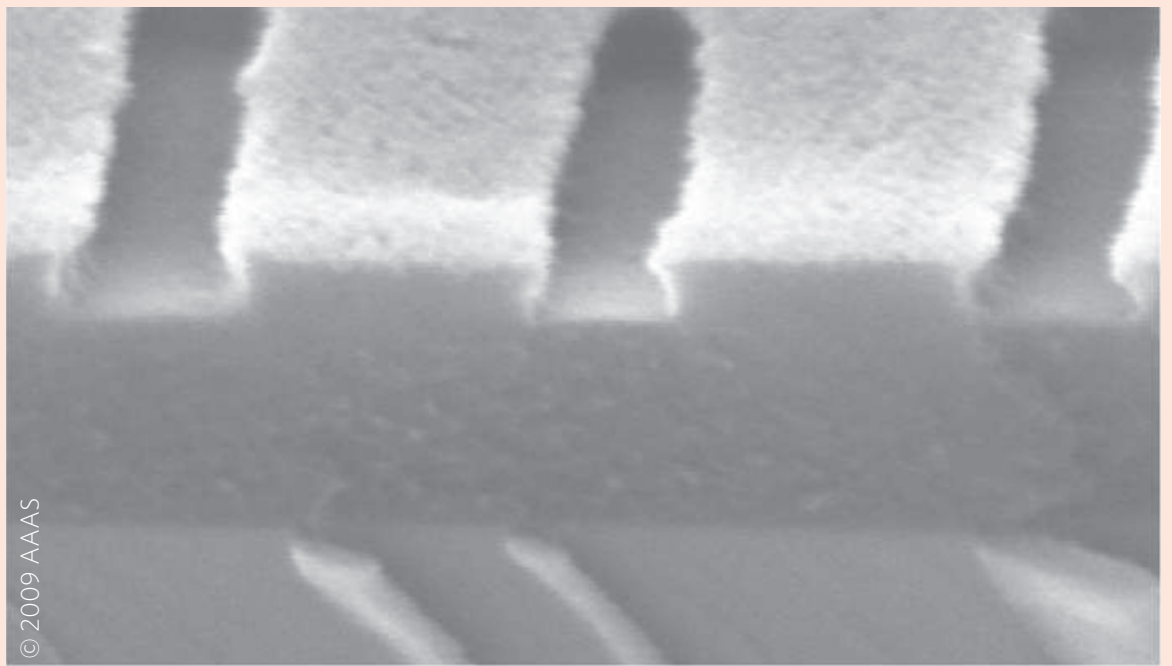

(Science doi: 10.1126/science.1167610; 2009) also used a two-wavelength technique to perform subdiffraction photolithography in an approach based on photo-polymerization, while researchers at the University of Maryland (Science doi: 10.1126/science.1168996; 2009) used two lasers - one continuous and one pulsed - operating at a wavelength of $800 \mathrm{~nm}$ to produce feature sizes as small as $40 \mathrm{~nm}$, which is a factor of 20 less than the wavelength.

\section{PETER RODGERS}

